\title{
Closed-loop Neurostimulation: The Clinical Experience
}

\author{
Felice T. Sun • Martha J. Morrell
}

Published online: 22 May 2014

(C) The Author(s) 2014. This article is published with open access at Springerlink.com

\begin{abstract}
Neurostimulation is now an established therapy for the treatment of movement disorders, pain, and epilepsy. While most neurostimulation systems available today provide stimulation in an open-loop manner (i.e., therapy is delivered according to preprogrammed settings and is unaffected by changes in the patient's clinical symptoms or in the underlying disease), closed-loop neurostimulation systems, which modulate or adapt therapy in response to physiological changes, may provide more effective and efficient therapy. At present, few such systems exist owing to the complexities of designing and implementing implantable closed-loop systems. This review focuses on the clinical experience of four implantable closed-loop neurostimulation systems: positional-adaptive spinal cord stimulation for treatment of pain, responsive cortical stimulation for treatment of epilepsy, closed-loop vagus nerve stimulation for treatment of epilepsy, and concurrent sensing and stimulation for treatment of Parkinson disease. The history that led to the development of the closed-loop systems, the sensing, detection, and stimulation technology that closes the loop, and the clinical experiences are presented.
\end{abstract}

Key Words Responsive $\cdot$ adaptive $\cdot$ stimulation $\cdot$ pain . epilepsy · Parkinson disease

Electronic supplementary material The online version of this article (doi:10.1007/s13311-014-0280-3) contains supplementary material, which is available to authorized users.

F. T. Sun $(\bowtie) \cdot$ M. J. Morrell

NeuroPace Inc., 445 N. Bernardo Avenue, Mountain View, CA 94043, USA

e-mail: fsun@neuropace.com

M. J. Morrell

Stanford University, Neurology, Stanford, CA, USA

\section{Introduction}

In the last 2 decades, since the US Food and Drug Administration (FDA) approval of deep brain stimulation (DBS) for the treatment of tremor in 1997 [1], neurostimulation has become an important therapeutic option for patients with diseases of the nervous system. Neurostimulation is now an established therapy for treatment of essential tremor [2], Parkinson disease (PD) [3, 4], epilepsy [5, 6], and neuropathic pain $[7,8]$, and is being investigated for numerous other neurologic and psychiatric disorders, including memory disorders, depression, obsessive-compulsive disorder, and Tourette syndrome $[9,10]$.

Most neurostimulation systems available today provide stimulation in an open-loop manner, which means that stimulation settings are preprogrammed and do not automatically respond to changes in the patient's clinical symptoms or in the underlying disease. While open-loop stimulation paradigms are effective, limitations of open-loop stimulation have become more evident as clinical experience grows. For example, although open-loop spinal cord stimulation systems are generally effective for the treatment of pain, open-loop systems may provide too much or too little therapy because the stimulation settings are not automatically adjusted based on the patient's body position [11-13]. A closed-loop system may provide improved and more consistent pain relief by automatically adjusting the stimulation settings according to the patient's body position. In another example, open-loop DBS systems for the treatment of $\mathrm{PD}$, while effective in managing the motor symptoms, may be inefficient because the same level of stimulation is provided regardless of the extent of motor impairment [14]. A closed-loop system may provide stimulation more efficiently by delivering stimulation only when motor function is impaired.

Advancements in implantable technology have led to the design and clinical introduction of implantable closed-loop 
neurostimulation systems that continuously sense physiological signals, detect prespecified physiological changes, and adjust therapy in response to the detected signals. Closedloop therapies may offer advantages relative to open-loop therapies by increasing the efficacy of stimulation [14-16], improving the clinical benefit of stimulation [16], and reducing the side effects of stimulation [14].

This review focuses on the clinical experience of implantable closed-loop neurostimulation systems for treatment of pain, epilepsy, and movement disorders. The history that led to the development of the closed-loop system, the sensing, detection, and stimulation technology that closes the loop, and the clinical experience are presented.

\section{Pain}

Background

Neurostimulation to treat pain was introduced clinically in 1967, when the first spinal cord stimulator was implanted to stimulate the dorsal columns of the spinal cord [17]. Since then, numerous technological developments, including improved lead design and advanced programming capabilities, have improved the efficacy of the therapy. Spinal cord stimulation (SCS) is now a widely accepted form of therapy for chronic intractable neuropathic pain and is the most commonly employed neurostimulation therapy for treatment of pain [18].

SCS provides pain relief by interfering with pain signals traveling along the spinal cord. To achieve optimum efficacy, the stimulation settings must be adjusted for each patient. Stimulation amplitude that is too low may be ineffective, and stimulation amplitude that is too high may be perceived as painful [11].

One of the challenges of SCS systems is to provide effective pain relief in all body positions. Because the stimulating electrodes are typically situated in the epidural space, a change in body position may change the epidural distance or the longitudinal distance, which may result in a change in the location and intensity of the stimulation-induced effect [11-13, 19]. For example, the same stimulation settings that are effective when the patient is standing may be too intense when the patient is lying down.

Open-loop SCS systems address this issue using a patientcontrolled programmer that allows the patient to adjust the stimulation intensity within clinician-prescribed limits. However, the patient may need to make multiple adjustments during the day to maintain adequate pain control. Recently, a closed-loop SCS system, the RestoreSensor system (Medtronic, Minneapolis, MN, USA), has been approved by the US FDA to provide automatic adjustments in stimulation according to the patient's body position (Fig. 1).

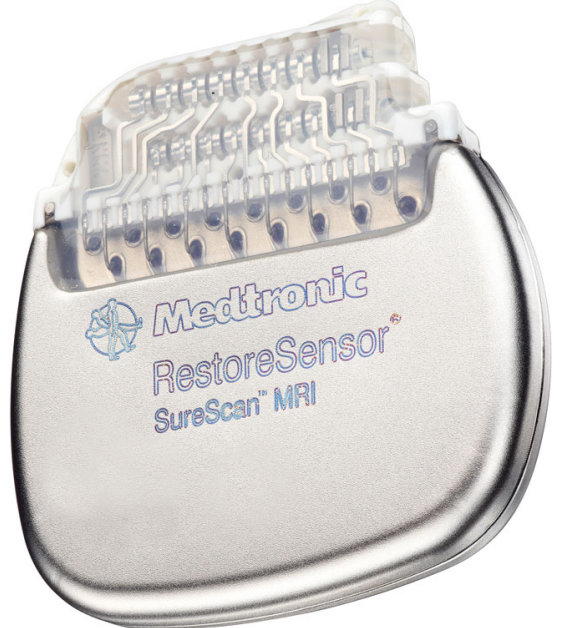

Fig. 1 The Medtronic RestoreSensor SureScan MRI SCS device (Medtronic, Minneapolis, MN, USA). Reprinted with the permission of Medtronic, Inc. (C) 2013

\section{Closed-loop Technology}

The RestoreSensor system uses a 3-axis accelerometer that senses the patient's body position and activity, and then adjusts the stimulation settings according to the body position. In order to provide this closed-loop therapy, the RestoreSensor neurostimulator must first be trained to recognize different body positions. During this process, the patient is positioned in multiple orientations such as upright, lying prone, lying supine, lying on the left side, and lying on the right side. Data from the 3 -axis accelerometer are linked to each of these positions, and patient-specific stimulation settings are optimized for each position.

Once the position-adaptive stimulation feature is programmed, the system detects changes in body position and activity in real time and automatically adjusts stimulation according to the preprogrammed settings.

\section{Clinical Experience}

Two clinical studies assessed safety and efficacy of closedloop SCS for pain $[16,20]$.

The first evaluated the feasibility and utility of an accelerometer-based algorithm to automatically adjust stimulation settings based on body position or activity [20]. This prospective, open-label, randomized study enrolled 20 patients across 2 centers. Fifteen participants completed the in-clinic protocol to provide data for the study.

Prior to entering this study, patients had already been implanted with either the Restore or RestoreAdvanced spinal cord stimulator (Medtronic) for at least 3 months, demonstrated stable pain control with their neurostimulation system, and used their patient programmer to change the stimulation 
amplitude in response to body position or activity at least twice a day during a 3-day baseline period.

During the in-clinic phase, the patient's baseline stimulation settings were recorded. Additionally, stimulation thresholds were obtained for 8 different positions: standing, sitting, lying supine, lying on right side, lying prone, lying on left side, reclining at $45^{\circ}$, and walking on a treadmill. An external sensor containing a triaxial accelerometer was then fitted to the patient, and accelerometer measurements were taken for a subset of those positions. The external sensor communicated directly with a programmer to adjust the stimulation settings according to the measured body position.

Each patient self-rated their overall satisfaction for each of the 8 positions when stimulation was adjusted manually and with two different automatic stimulation adjustment modes. Patients reported significantly higher satisfaction using the automatic stimulation adjustment modes compared with the manual adjustment mode, and $74 \%$ of patients reported that stimulation settings using the automatic adjustment algorithm were "just right" ( $v s$ "too high" or "too low"). This study demonstrated the feasibility and utility of using a triaxial accelerometer to measure the body position in order to automatically adjust the stimulation settings.

The automatic adjustment algorithm was subsequently evaluated in a clinical study of an implantable spinal cord neurostimulation system [16]. A prospective, multicenter, open-label, randomized crossover study was conducted in 79 patients implanted with the RestoreSensor (Medtronic) SCS system to assess whether the position-adaptive stimulation feature provided benefit in terms of pain relief and/or convenience compared with not using the feature.

For the first 4 weeks after implantation of the system, only manual adjustment of stimulation was enabled. Patients were then randomized 1:1 to receive either position-adaptive stimulation or conventional manual programming for 6 weeks, and were then crossed over to receive the other type of stimulation for 6 weeks.

In the intent-to-treat analysis, $86.5 \%$ of patients achieved the primary objective of improved pain relief with no loss of convenience, or had improved convenience with no loss of pain relief using automatic position-adaptive stimulation compared with using conventional manual programming adjustment alone. Moreover, patients reported improved comfort during position changes $(80.3 \%)$, improved activity $(69.0 \%)$, and improved sleep (47.9 \%) with positionadaptive stimulation. There were 25 adverse events (AEs) related to undesirable changes in stimulation; however, only 9 of the events occurred during the position-adaptive stimulation arm of the study [16].

These studies illustrate how a fairly straightforward and simple closed-loop system using body orientation as a feedback signal significantly improved the patient experience. Closed-loop position-adaptive stimulation improved patient- reported pain relief, activity, sleep, and convenience compared with using manual programming adjustment alone. Future SCS systems may employ more sophisticated closed-loop algorithms, such as using electrically evoked compound action potentials to automatically adjust stimulation parameters, which could allow for even more effective and efficient pain control [21].

\section{Epilepsy}

The earliest report of applying electrical stimulation to the brain to treat seizures in humans is by Penfield and Jasper in 1954 [22]. In their acute experiments, they observed that in some cases electrical stimulation of the cortex resulted in a flattening of the local electrocorticogram (both normal rhythms and spontaneous epileptiform discharges).

Numerous studies published over the last 6 decades have evaluated the safety and efficacy of brain stimulation to treat epilepsy [23]. Promising results were reported for stimulation of the cerebellum [24-26], caudate nucleus [27-29], centromedian nucleus $[28,30,31]$, subthalamic nucleus (STN) [32], hippocampus [33-35], and the anterior nucleus of the thalamus [36-38]. However, with the exception of a randomized double-blinded controlled trial of stimulation of the anterior nucleus of the thalamus as adjunctive treatment of medically intractable partial onset seizures in adults [38], these studies were largely uncontrolled, small, and have not been replicated.

There are currently 2 neurostimulation therapies approved by the US FDA for treatment of epilepsy: open-loop vagus nerve stimulation (VNS Therapy Cyberonics, Houston, TX, USA) and closed-loop responsive cortical stimulation (RNS System, NeuroPace, Mountain View, CA, USA).

The following two sections describe the background, the closed-loop technology, and clinical experience of the US FDA-approved closed-loop responsive cortical stimulation system and an investigational closed-loop vagus nerve stimulation system, which builds on the approved open-loop vagus nerve stimulation technology.

\section{Closed-loop Responsive Cortical Stimulation}

\section{Background}

Initial experiments assessed the safety and efficacy of responsive electrical stimulation for epilepsy during in-patient intracranial monitoring to determine whether the patient was a candidate for epilepsy surgery. A routine procedure during this evaluation is to deliver focal cortical stimulation to identify functional and epileptogenic regions. At times, the electrical stimulation elicits afterdischarges (ADs), which are rhythmic discharges that are similar to spontaneous 
epileptiform activity. Lesser et al. [39] observed that a brief burst of electrical stimulation (similar to the stimulation used to elicit $\mathrm{ADs}$ ) could also terminate an $\mathrm{AD}$. The most effective stimulation to terminate ADs was brief $(0.5-1 \mathrm{~s})$, applied early (within $4.5 \mathrm{~s}$ of the AD), and applied at the site of the $\operatorname{AD}[39,40]$.

This work paved the way for the development of closedloop responsive stimulation systems designed to deliver electrical stimulation in response to spontaneous epileptiform activity. The first such systems were large, nonimplantable systems, developed as proof-of-concept prototypes to assess whether closed-loop responsive stimulation was feasible and could be effective [41, 42].

Peters et al. [41] used an external bedside system that performed real-time seizure detection and automatically delivered electrical stimulation in response to the seizure detection. This system was evaluated in 8 patients undergoing intracranial monitoring [43]. Four patients received responsive stimulation directly to the epileptogenic zone (local closed-loop) and 4 received responsive stimulation to the anterior thalami (remote closed-loop). The mean reduction in seizures in the local closed-loop stimulation group was $55.5 \%$ and $40.8 \%$ in the remote closed-loop group. Four of the 8 patients had $a \geq 50 \%$ reduction in seizures with responsive stimulation (3 with local closed-loop stimulation and 1 with remote closed-loop stimulation). Kossoff et al. [42] also reported on the experience using an external responsive neurostimulator in 4 patients. Stimulation delivered in response to epileptiform activity appeared to reduce the number of clinical seizures and to suppress electrographic seizures. These studies demonstrated that closed-loop responsive stimulation was feasible and provided preliminary evidence that responsive stimulation could reduce seizures.

\section{Closed-loop Technology}

The RNS System is a responsive cortical neurostimulator system approved by the US FDA as an adjunctive therapy in reducing the frequency of seizures in individuals aged 18 years or older with partial onset seizures who have undergone diagnostic testing that localized no more than 2 epileptogenic foci, are refractory to $\geq 2$ antiepileptic medications, and currently have frequent and disabling seizures (motor partial seizures, complex partial seizures, and/or secondarily generalized seizures). The RNS System includes a neurostimulator that is implanted in the cranium and connected to 1 or 2 recording and stimulating depth and/or cortical strip leads that are surgically placed in the brain at the seizure foci (Fig. 2). The closed-loop neurostimulator provides responsive electrical stimulation directly to 1 or 2 seizure foci when abnormal electrocorticographic activity is detected. The system includes a programmer for the physician, a remote monitor for the patient, and a secure internet-accessed database for storage

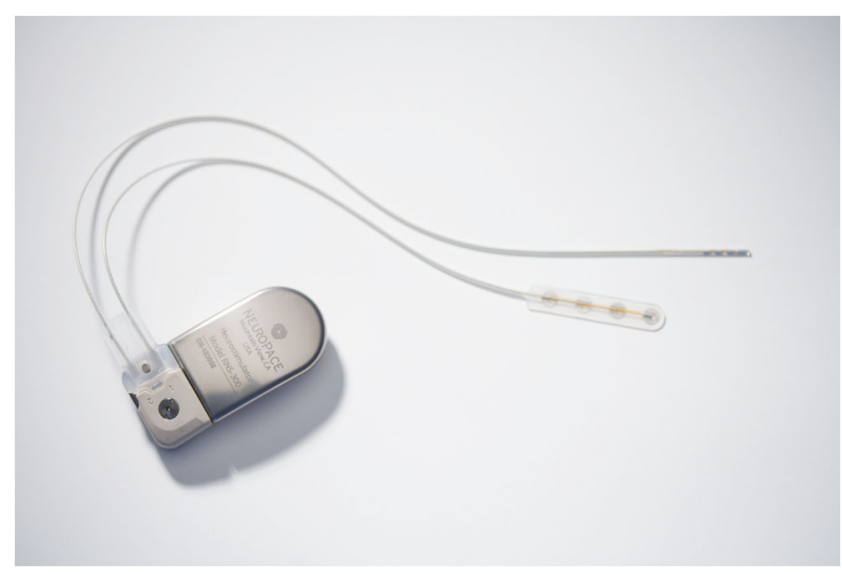

Fig. 2 The RNS Neurostimulator connected to the NeuroPace Depth Lead and NeuroPace Cortical Strip Lead (NeuroPace, Mountain View, CA, USA)

of neurostimulator data obtained by the programmer or remote monitor.

\section{Sensing and Data Storage}

The neurostimulator continuously senses and monitors electrographic activity through the implanted cortical depth and strip leads. The neurostimulator records the date and time of all detections and stimulations, and stores segments of the electrographic data for review by the physician. Electrographic data storage is triggered by detection, responsive stimulation, scheduled time of the day, magnet (used by the patient to indicate a seizure), and/or other events as programmed by the physician. These data allow physicians to assess detection sensitivity and effects of stimulation.

\section{Detection}

The detection algorithms in the neurostimulator are computationally efficient and optimized in order to perform real-time detection within the constraints of currently available implantable technology, such as limited power and processing capabilities [44]. Three detection tools (bandpass, line-length, and area) are provided. The detection parameters are highly configurable and are selected by the physician to adjust the sensitivity, specificity, and latency of the detection.

The bandpass tool is similar to that described by Gotman [45], and is used to detect spikes and rhythmic activity occurring in specific frequency ranges. This tool uses "half-waves", which are segments of the electrographic signal partitioned at local minima and maxima. The amplitude and duration of the half-waves are representative of the amplitude and frequency components of the electrographic signal.

The line-length algorithm, described by D'Alessandro et al. [46] and Esteller et al. [47] identifies changes in both 
amplitude and frequency. The line-length is defined as the average of absolute sample-to-sample amplitude differences within a window. A short-term sliding window average (128 ms-4 s) is compared with a long-term sliding window average (4 s-16 min). Detection occurs when the short-term measurement crosses an absolute or relative threshold derived from the long-term measurement.

The area feature is similar to an energy or power measurement and identifies changes in overall signal energy without regard for frequency [48-50]. Area is defined as the average absolute area-under-the-curve of the electrographic signal. As with line-length, a short-term window average is compared with a long-term background window average, and detection occurs when a previously defined threshold is crossed.

These 3 detection algorithms are efficient (requiring low computational power), and can be configured to detect electrographic events within a fraction of a second or to detect more subtle changes in amplitude, frequency, and/or power that occur over several seconds.

\section{Stimulation}

The neurostimulator delivers current-controlled, chargebalanced biphasic pulses, and is programmed by the physician to deliver stimulation frequencies ranging from 1 to $333 \mathrm{~Hz}$, current amplitudes from 0.5 to $12.0 \mathrm{~mA}$, and pulse-widths from 40 to $1000 \mu \mathrm{s}$. The stimulation montage can be configured to deliver current between any combination of electrodes, including the neurostimulator case.

\section{Clinical Use}

The RNS System is not a seizure predictor and cannot determine if the patient has a clinical seizure. In the clinical experience to date, the neurostimulator has typically been programmed to detect spike and slow waves, rhythmic changes in frequency, or changes in amplitude typical of the electrocorticographic features that sometimes progressed to an electrographic and/or clinical seizure. The most common stimulation programming was an amplitude of $1.5-3.0 \mathrm{~mA}$, a pulse width of $160 \mu$ s, a stimulation burst duration of 100 $200 \mathrm{~ms}$, and a pulse frequency of between 100 and $200 \mathrm{~Hz}$. The majority of patients received stimulation in response to detections 600-2000 times a day for a cumulative total of $<5$ mins of stimulation over $24 \mathrm{~h}$. As this closed-loop stimulation was in response to detections of specific electrocorticographic patterns, and it was not possible to know which of these detections would have progressed to an electrographic (or clinical) seizure, the concept of false-positive or falsenegative seizure detections was therefore not relevant to this stimulation approach.

\section{Clinical Experience}

Safety and efficacy of the RNS System as an adjunctive treatment in adults with medically intractable partial-onset seizures was established in 3 clinical trials: a 2 -year primarily open-label safety study (feasibility study, $n=65$ ), a 2 -year double-blinded randomized sham-stimulation controlled study (pivotal study, $n=191$ ), and a long-term extension study (long-term treatment study) designed to collect an additional 7 years of efficacy and safety data in patients completing the feasibility or pivotal studies.

The pivotal study was a multicenter, double-blinded, randomized sham-stimulation controlled study to demonstrate the safety and effectiveness of the RNS System as an adjunctive treatment for adults with medically intractable partialonset seizures arising from 1 or 2 seizure foci. Patients were, on average, 34.9 years old and had epilepsy for 20.5 years. Nearly one-third ( $32 \%$ ) had prior therapeutic epilepsy surgery (resection, subpial transection, and/or callosotomy), and more than a third (34\%) had previously been treated with a vagus nerve stimulator.

The effectiveness of responsive stimulation was assessed by comparing the seizure reduction in the group receiving active stimulation (treatment group) versus the group receiving no stimulation (sham group) during a 12-week blinded period relative to a preimplant baseline. The primary effectiveness endpoint was met: the reduction in seizure frequency in the treatment group $(-37.9 \%)$ was significantly greater than that in the Sham group $(-17.3 \% ; p=0.012)$ [6]. There was no difference in effectiveness in patients with mesial temporal lobe seizure onsets compared with patients with neocortical seizure onsets, in patients whose seizures arose from $1 \mathrm{com}$ pared with 2 foci, in patients who had been treated with vagus nerve stimulation (VNS) compared with those who had not, and in patients who had already undergone a therapeutic epilepsy surgery compared with those who had not.

During the open-label period of the study, when all patients had the opportunity to receive responsive stimulation, seizure reduction continued to improve. The median percent reduction in seizures was $44 \%$ at 1 year and $53 \%$ at 2 years postimplant compared with baseline [51].

Safety was assessed using AE data. The RNS System serious $\mathrm{AE}$ rate was no worse than the literature-derived serious AE rate for comparable procedures. Stimulation was well tolerated. There was no difference in the frequency or type of AE between the treatment and sham groups, except for side effects of antiepileptic medications, which were more common in the sham group (5 patients, all mild events) than the treatment group (none). (Refer to the RNS System product labeling for detailed disclosure of specific indications, contraindications, warnings, precautions, and AEs.)

Additional assessments included a quality of life inventory, neuropsychological evaluations, and mood inventories. At 1 
and 2 years after implantation, patients reported significant improvements in overall quality of life $(p<0.001)$ and in 9 of the primary scale scores, including memory, concentration, and language [51]. There was no significant deterioration in any neuropsychological measures or any of the mood inventories at the end of the blinded period compared with the baseline period, or at 1 and 2 years postimplant, indicating there were no acute, delayed, or longer-term adverse effects of responsive stimulation on neuropsychological function and mood.

These results demonstrate that closed-loop responsive stimulation to the seizure focus can reduce the frequency of partial onset seizures, is well-tolerated, and is acceptably safe.

\section{Closed-loop VNS}

\section{Background}

Open-loop VNS is approved by the US FDA for use as an adjunctive therapy in reducing seizure frequency in adults and adolescents over the age of 12 years with partial-onset seizures that are refractory to antiepileptic drugs. Intermittent scheduled stimulation is delivered to the vagus nerve. A typical stimulation schedule is $30 \mathrm{~s}$ "on" and $5 \mathrm{~min}$ "off," but this can be adjusted by the physician. By moving a magnet over the VNS pulse generator, patients may also initiate on-demand stimulation bursts to provide additional VNS therapy during an aura or at the onset of a seizure.

Two randomized, blinded, active-control (high-stimulation/low-stimulation) trials evaluated the safety and efficacy of open-loop VNS therapy [5, 52]. The median percent reduction in daily seizures was $23-24 \%$ in the high stimulation group and 6-21\% in the low stimulation group. The reduction in seizures in an open-label extension study was $31 \%$ at 1 year and $41 \%$ at 2 years. (Refer to the VNS therapy product labeling for detailed disclosure of specific indications, contraindications, warnings, precautions, and AEs.)

The concept of closed-loop VNS therapy emerged from the experience with magnet-activated stimulation, where a magnet is used by the patient or caregiver to trigger additional bursts of stimulation at the time of an aura or seizure onset. One prospective and 1 retrospective study evaluated magnetactivated VNS therapy $[53,54]$. The first was a single-center, prospective study of 35 patients that was designed to assess the efficacy of magnet-activated stimulation [53]. After implantation of the VNS system, patients and their caregivers were provided with a magnet and instructed how to use the magnet to provide additional stimulation when an aura or seizure onset occurred. Of the 35 implanted patients, 21 (or their caregivers) were able to use the magnet and provide reliable seizure information. Fourteen of the 35 patients were unable to use the magnet: 9 because they had no auras or their seizures were too brief; 3 had become seizure-free before the magnet was provided; and 2 had unreliable data. Of the 21 patients who were able to use the magnet, 14 reported a positive effect of the magnet and 7 reported no effect of the magnet. Of note, only 3 patients were able to use the magnet themselves. In most cases, support from caregivers was necessary. Data from this study suggest that acute VNS delivered at the onset of a seizure may provide added benefit to the standard scheduled VNS, but reliable manual delivery of stimulation may be difficult to achieve.

A second, retrospective study evaluated magnet usage during two open-loop VNS trials: the E03 and E04 trials [54]. In the randomized, double-blind, controlled, E03 trial, patients were randomized 1:1 to receive therapeutic VNS therapy (treatment group) or nontherapeutic stimulation (active control group). During the blinded period of the trial, magnet-activated stimulation was on for the treatment group and off for the active control group. Of the 114 participants in the E03 study, 92 (or their caregivers) used the magnet (50 in the treatment group and 42 in the active control group). The treatment group reported that $21.3 \%$ of the seizures that received magnet-activated stimulation were terminated compared with $11.9 \%$ in the active-control group $(p=0.08)$. Additionally, the treatment group was more likely to report improvement with magnet usage compared with the active control group $(p=0.05)$.

During the open-label E04 trial, 86/124 participants used their magnets. Of the 86 patients (or caregivers) who used the magnets, $22 \%$ reported seizure termination, $31 \%$ reported seizure diminution, and $47 \%$ reported no effect of magnetactivated VNS therapy. Patient-reported outcomes were available for 9482 seizures for which magnet-activated VNS was used: for 2211 seizures ( $24 \%$ ) magnet-activated VNS terminated the event, for 3638 seizures (38\%) magnet-activated VNS diminished the event, and for 3633 seizures (38\%) magnet-activated VNS did not affect the event.

Results from both studies of magnet-activated stimulation suggest that there could be a positive effect of additional VNS therapy during an aura or at the onset of a seizure. However, both studies also revealed that most patients were unlikely to be able to use the magnet on their own - a caregiver was typically involved in activating the additional stimulation. This led to the development of a closed-loop VNS system, the AspireSR (Cyberonics), which is an investigational vagus nerve stimulator that automates the delivery of additional stimulation using a cardiac-based seizure detector.

\section{Closed-loop Technology}

Cardiac-based seizure detection relies on changes in the heart rate at the onset or during a seizure. The most frequently reported type of seizure-related cardiac change is ictal tachycardia, or an increase in heart rate with seizure, which occurs in $>70 \%$ of seizures [55-58]. Thus, several groups have 
proposed cardiac-based seizure detection based on this increase in heart rate [59-62].

Although the cardiac-based seizure detection algorithm for the AspireSR generator has not been published, one computationally efficient approach that could be performed in realtime in an implantable system is to track the heart rate using both long- and short-term trends [59]. The long-term heart rate trend represents the background rate, which may change slowly over time based on the patient's activity level. The short-term heart rate trend represents the foreground rate. When the foreground heart rate exceeds a threshold relative to the background heart rate, an event is detected. Because the threshold is based on the background heart rate, it automatically adjusts to the patient's underlying activity.

\section{Clinical Experience}

The AspireSR is a vagus nerve stimulator with a cardiac-based seizure detection feature. The performance of the cardiacbased seizure detection feature was evaluated in a study of 31 patients. Patients implanted with the AspireSR generator were observed in epilepsy monitoring units for up to 5 days to identify seizures and collect heart rate data. More than $80 \%$ of seizures that were accompanied by ictal tachycardia were detected by the AspireSR generator. Thus, the primary endpoint of the study was met. The potential false detection rates were low. The detections occurred close, and in some cases prior, to seizure onset. This study demonstrated that the cardiac-based seizure detection algorithm implemented in the AspireSR system could detect seizures with heart rate changes [63].

\section{Movement Disorders}

\section{Background}

DBS has been a treatment option for movement disorders since 1997 when the first DBS system was approved for treatment of tremor [1]. Over the last 2 decades, open-loop DBS has proven to be a valuable and effective therapy option for patients with PD, essential tremor, or dystonia whose motor symptoms cannot be controlled by drug therapy alone [3, 4, 64-67].

Despite the success of open-loop DBS for movement disorders, many believe that closed-loop technology can improve the therapy by potentially reducing the side effects of stimulation and/or improving the efficacy of stimulation. For example, side effects associated with DBS such as impairment of speech, gait, and balance may be ameliorated by a closed-loop responsive stimulation approach where stimulation is delivered intermittently [14, 68, 69]. Additionally, closed-loop responsive or adaptive stimulation may improve the efficacy of DBS [14, 15]. Indeed, Rosin et al. [15] discovered that delivering closed-loop stimulation to the internal segment of the globus pallidus in response to action potentials recorded in the primary motor cortex is more efficient and effective in alleviating Parkinsonian motor symptoms than continuous stimulation.

There are also other potential advantages of closed-loop stimulation. Closed-loop responsive stimulation may increase the battery longevity, thus exposing the patient to fewer neurostimulator replacement procedures. Additionally, closed-loop systems may facilitate the process of identifying the optimal stimulation settings by using a physiological signal as a biomarker to titrate the stimulation parameters, rather than relying on clinically overt symptoms [70, 71]. Thus, there is significant interest in developing closed-loop DBS systems for treatment of movement disorders.

A number of approaches have been proposed using various physiological signals for feedback in the closed-loop system, including electromyography [72-74] and single- or multi-unit recordings [15]. However, these signals are not amenable to chronic recording. Perhaps the most practical approach is to leverage existing open-loop neurostimulation systems and use local field potentials (LFPs) sensed from the stimulating electrode to close the loop $[14,75]$.

For treatment of PD, several groups have proposed using the power in the beta frequency band $(13-30 \mathrm{~Hz})$ of the LFP in the STN as a biomarker for closed-loop stimulation [76-78]. In patients with $\mathrm{PD}$, there is often a prominence in the beta frequency band that is correlated with the severity of motor symptoms and is suppressed with levodopa treatment [79], as well as with DBS $[76,79,80]$. Moreover, the suppression of the beta activity is correlated with improved motor performance [76]. Therefore, the beta band may be a useful biomarker for the severity of motor symptoms and the efficacy of stimulation.

Little et al. [14] provided a proof-of-principle of this approach. In 8 patients undergoing DBS implantation for PD, the LFP recorded from the stimulating electrode implanted in the STN was used to trigger closed-loop stimulation. Specifically, the LFP was filtered and rectified to produce an "online" value of beta amplitude. This signal was used to trigger stimulation via a user-defined threshold such that stimulation was delivered approximately $50 \%$ of the time. Stimulation, once triggered, was sustained until the beta amplitude fell below the threshold. The authors reported that this closed-loop stimulation resulted in improved motor response relative to continuous open-loop stimulation. This research demonstrates that the LFP in the STN could be a useful biomarker to control DBS.

\section{Closed-loop Technology}

A fully implantable closed-loop DBS system has recently been developed for investigational use. This system, the Activa $\mathrm{PC}+\mathrm{S}$ neurostimulator (Medtronic), is based on the 
Activa PC neurostimulator with additional sensing, stimulation, and detection features (Fig. 3).

The main technical challenge in designing a closed-loop concurrent sensing and stimulation system, in which sensing is performed through the same leads as stimulation, is in separating the stimulation signal (artifact) from the signal of interest. Stanslaski et al. [81, 82] describe a systems-level approach to minimizing the stimulation interference. This approach includes design elements in the sensing component, the stimulation component, and the detection algorithm component.

\section{Sensing}

Three key design elements in the sensing component contribute to the elimination of the stimulation artifact. First, by using differential sensing and electrodes that are symmetric in geometry about the stimulating electrode, a large component of the stimulation artifact can be rejected as a common mode disturbance. Second, a front-end filter can be configured to suppress the high frequency associated with stimulation. For example, a stimulation frequency of $140 \mathrm{~Hz}$ can be suppressed with a $100-\mathrm{Hz}$ low-pass filter. Third, a spectral bandpower processor can be used to isolate the signal power in the specific frequency band of interest, thus separating the neural biomarkers from the stimulation harmonics.

\section{Stimulation}

While the design elements in the sensing component are key to suppressing the stimulation artifact in the frequency band of interest, careful selection of the sampling rate and the

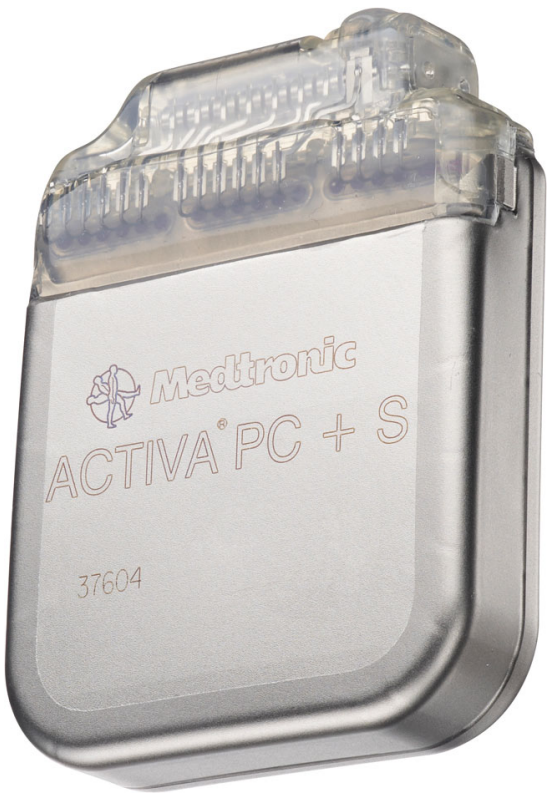

Fig. 3 The Activa ${ }^{\mathbb{R}} \mathrm{PC}+\mathrm{S}$ deep brain neurostimulator (Medtronic, Minneapolis, MN, USA). Reprinted with the permission of Medtronic, Inc. (C) 2013 stimulation frequency are also necessary to separate the stimulation artifact from the signal of interest. Although the stimulation frequency may be higher than the frequency band of interest, the stimulation frequency and its harmonics may be aliased back into lower frequency bands depending on the sampling rate. Therefore, the design includes numerical analysis methods to identify stimulation frequencies that minimize the interference with the band of interest based on the sampling rate.

\section{Detection Algorithm}

Finally, the design includes detection algorithms that further distinguish the information in the signal of interest from the stimulation signal. For example, supplementing the physiological spectral channels with independent information about the stimulation energy may allow a classification algorithm such as a support vector machine to better separate the stimulation signal from the signal of interest.

These design elements, as described by the authors "with modest performance in each individual block but acceptable overall performance" enable measurement of the LFP in the STN during stimulation, thus paving the way for closed-loop DBS.

Proof-of-concept of the concurrent sensing and stimulation design was demonstrated in a large animal (ovine) model with leads implanted in the thalamus and hippocampus [71, 81]. Evoked potentials and LFPs could be recorded during stimulation. Moreover, the evoked potentials were stable for $>1$ year, demonstrating the utility of the system as a chronic implant [71].

\section{Clinical Experience}

The investigational Activa $\mathrm{PC}+\mathrm{S}$ DBS is currently being used in a clinical trial to study changes in neuronal oscillations during tremor, repetitive movement, and freezing episodes relative to rest in patients with $\mathrm{PD}$, with the first human implants in 2013 [83]. There have been no published reports on experience with the system.

\section{Discussion}

We have reviewed 4 implantable closed-loop neurostimulation systems: positional-adaptive SCS for treatment of pain, responsive cortical stimulation for the treatment of epilepsy, closedloop VNS for the treatment of epilepsy, and concurrent sensing and stimulation for the treatment of PD. At present, few such systems exist owing to the complexities of designing and implementing such a system. The technical challenges include incorporating physiological sensors that add minimal risk to the patient, and developing algorithms that detect in real-time and 
require low computational power. The scientific and clinical challenges include determining the physiological markers for specific symptoms, the anatomical target in which to sense and stimulate, the physiological changes to detect, and how to deliver and modulate the stimulation in response to the detected event and, ultimately, the clinical symptoms. However, the clinical experience from these systems support the notion that closed-loop therapy can be more effective than open-loop ones, and therefore underscores the importance of continued effort in developing closed-loop systems and in identifying promising clinical applications. Future applications of closed-loop neurostimulation may include treatment of major depression [84], Tourrette syndrome [85], and other neuropsychiatric disorders. The success of closed-loop SCS for the treatment of pain and closed-loop responsive cortical stimulation for treatment of epilepsy pave the way for development of these other closed-loop neurostimulation systems.

Required Author Forms Disclosure forms provided by the authors are available with the online version of this article.

Open Access This article is distributed under the terms of the Creative Commons Attribution License which permits any use, distribution, and reproduction in any medium, provided the original author(s) and the source are credited.

\section{References}

1. FDA. Medtronic Activa tremor control system P960009. Available at: http://www.accessdata.fda.gov/cdrh docs/pdf/p960009.pdf. Accessed Feb 19, 2014.

2. Lyons KE, Pahwa R. Deep brain stimulation and essential tremor. J Clin Neurophysiol 2004;21:2-5.

3. Weaver FM, Follett K, Stern M, et al. Bilateral deep brain stimulation vs best medical therapy for patients with advanced Parkinson disease: a randomized controlled trial. JAMA 2009;301:63-73.

4. Deuschl G, Schade-Brittinger C, Krack P, et al. A randomized trial of deep-brain stimulation for Parkinson's disease. N Engl J Med 2006;355:896-908.

5. Handforth A, DeGiorgio CM, Schachter SC, et al. Vagus nerve stimulation therapy for partial-onset seizures: a randomized activecontrol trial. Neurology 1998;51:48-55.

6. Morrell MJ, RNS System in Epilepsy Study Group. Responsive cortical stimulation for the treatment of medically intractable partial epilepsy. Neurology 2011;77:1295-1304.

7. Kumar K, North R, Taylor R, et al. Spinal cord stimulation vs. conventional medical management: A Prospective, randomized, controlled, multicenter study of patients with failed back surgery syndrome (PROCESS Study). Neuromodulation 2005;8: 213-218.

8. Kumar K, Taylor RS, Jacques L, et al. The effects of spinal cord stimulation in neuropathic pain are sustained: a 24-month follow-up of the prospective randomized controlled multicenter trial of the effectiveness of spinal cord stimulation. Neurosurgery 2008;63: 762-770.

9. Laxton AW, Lipsman N, Lozano AM. Deep brain stimulation for cognitive disorders. Handb Clin Neurol 2013;116:307-311.
10. Hariz M, Blomstedt P, Zrinzo L. Future of brain stimulation: new targets, new indications, new technology. Mov Disord 2013;28:17841792.

11. Barolat G, Zeme S, Ketcik B. Multifactorial analysis of epidural spinal cord stimulation. Stereotact Funct Neurosurg 1991;56:77-103.

12. Cameron T, Alo KM. Effects of posture on stimulation parameters in spinal cord stimulation. Neuromodulation 1998;1:177-183.

13. Olin JC, Kidd DH, North RB. Postural changes in spinal cord stimulation perceptual thresholds. Neuromodulation 1998;1:171175.

14. Little S, Pogosyan A, Neal S, et al. Adaptive deep brain stimulation in advanced Parkinson disease. Ann Neurol 2013;74:449-457.

15. Rosin B, Slovik M, Mitelman R, et al. Closed-loop deep brain stimulation is superior in ameliorating parkinsonism. Neuron 2011;72:370-384.

16. Schultz DM, Webster L, Kosek P, et al. Sensor-driven positionadaptive spinal cord stimulation for chronic pain. Pain Physician 2012;15:1-12.

17. Shealy CN, Mortimer JT, Reswick JB. Electrical inhibition of pain by stimulation of the dorsal columns: preliminary clinical report. Anesth Analg 1967;46:489-491.

18. Cameron T. Safety and efficacy of spinal cord stimulation for the treatment of chronic pain: a 20 -year literature review. J Neurosurg 2004; 100:254-267.

19. Holsheimer J, den Boer JA, Struijk JJ, et al. MR assessment of the normal position of the spinal cord in the spinal canal. AJNR Am J Neuroradiol 1994;15:951-959.

20. Schade CM, Schultz DM, Tamayo N, et al. Automatic adaptation of neurostimulation therapy in response to changes in patient position: results of the Posture Responsive Spinal Cord Stimulation (PRS) Research Study. Pain Physician 2011;14: 407-417.

21. Parker JL, Karantonis DM, Single PS, et al. Compound action potentials recorded in the human spinal cord during neurostimulation for pain relief. Pain 2012;153:593-601.

22. Penfield W, Jasper H. Electrocorticography. Epilepsy and the functional anatomy of the human brain. Little, Brown, Boston, MA, 1954, pp. $692-738$.

23. Bergey GK. Neurostimulation in the treatment of epilepsy. Exp Neurol 2013;244:87-95.

24. Cooper IS, Amin I, Gilman S. The effect of chronic cerebellar stimulation upon epilepsy in man. Trans Am Neurol Assoc 1973;98:192-196.

25. Velasco F, Carrillo-Ruiz JD, Brito F, et al. Double-blind, randomized controlled pilot study of bilateral cerebellar stimulation for treatment of intractable motor seizures. Epilepsia 2005;46:1071-1081.

26. Krauss GL, Koubeissi MZ. Cerebellar and thalamic stimulation treatment for epilepsy. Acta Neurochir Suppl 2007;97: 347-356.

27. Chkhenkeli SA, Chkhenkeli IS. Effects of therapeutic stimulation of nucleus caudatus on epileptic electrical activity of brain in patients with intractable epilepsy. Stereotact Funct Neurosurg 1997;69:221224.

28. Chkhenkeli SA, Sramka M, Lortkipanidze GS, et al. Electrophysiological effects and clinical results of direct brain stimulation for intractable epilepsy. Clin Neurol Neurosurg 2004;106: 318-329.

29. Sramka M, Fritz G, Gajdosova D, et al. Central stimulation treatment of epilepsy. Acta Neurochir Suppl (Wien) 1980;30: 183-187.

30. Velasco F, Velasco M, Ogarrio C, et al. Electrical stimulation of the centromedian thalamic nucleus in the treatment of convulsive seizures: a preliminary report. Epilepsia 1987;28:421-430.

31. Fisher RS, Uematsu S, Krauss GL, et al. Placebo-controlled pilot study of centromedian thalamic stimulation in treatment of intractable seizures. Epilepsia 1992;33:841-851. 
32. Chabardes S, Kahane P, Minotti L, et al. Deep brain stimulation in epilepsy with particular reference to the subthalamic nucleus. Epileptic Disord 2002;4:S83-S93.

33. Vonck K, Boon P, Achten E, et al. Long-term amygdalohippocampal stimulation for refractory temporal lobe epilepsy. Ann Neurol 2002;52:556-565.

34. Tellez-Zentano JF, McLachlan RS, Parrent A, et al. Hippocampal electrical stimulation in mesial temporal lobe epilepsy. Neurology 2006;66:1490-1494.

35. Velasco AL, Velasco F, Velasco M, et al. Electrical stimulation of the hippocampal epileptic foci for seizure control: a double-blind, longterm follow-up study. Epilepsia 2007;48:1895-1903.

36. Cooper IS, Upton AR, Amin I. Reversibility of chronic neurologic deficits. Some effects of electrical stimulation of the thalamus and internal capsule in man. Appl Neurophysiol 1980;43:244-258.

37. Hodaie M, Wennberg RA, Dostrovsky JO, et al. Chronic anterior thalamus stimulation for intractable epilepsy. Epilepsia 2002;43:603608.

38. Fisher R, Salanova V, Witt T, et al. Electrical stimulation of the anterior nucleus of thalamus for treatment of refractory epilepsy. Epilepsia 2010;51:899-908.

39. Lesser RP, Kim SH, Beyderman L, et al. Brief bursts of pulse stimulation terminate afterdischarges caused by cortical stimulation. Neurology 1999;53:2073-2081.

40. Motamedi GK, Lesser RP, Miglioretti DL, et al. Optimizing parameters for terminating cortical afterdischarges with pulse stimulation. Epilepsia 2002;43:836-846.

41. Peters TE, Bhavaraju NC, Frei MG, et al. Network system for automated seizure detection and contingent delivery of therapy. J Clin Neurophysiol 2001;18:545-549.

42. Kossoff EH, Ritzl EK, Politsky JM, et al. Effect of an external responsive neurostimulator on seizures and electrographic discharges during subdural electrode monitoring. Epilepsia 2004;45:1560-1567.

43. Osorio I, Frei MG, Sunderam S, et al. Automated seizure abatement in humans using electrical stimulation. Ann Neurol 2005;57:258268.

44. Sun FT, Morrell MJ, Wharen RE, Jr. Responsive cortical stimulation for the treatment of epilepsy. Neurotherapeutics 2008;5:68-74.

45. Gotman J. Automatic recognition of epileptic seizures in the EEG. Electroencephalogr Clin Neurophysiol 1982;54:530-540.

46. D'Alessandro M, Esteller R, Vachtsevanos G, et al. Epileptic seizure prediction using hybrid feature selection over multiple intracranial EEG electrode contacts: a report of four patients. IEEE Trans Biomed Eng 2003;50:603-615.

47. Esteller R, Echauz J, Tcheng T, et al. Line length: An efficient feature for seizure onset detection. engineering in medicine and biology. Proceedings of the 23rd Annual International Conference of the IEEE 2001;2:1707-1710.

48. Echauz J, Padovani DA, Esteller R, et al. Median-based filtering methods for EEG seizure detection. Engineering in Medicine and Biology, 1999. 21st Annual Conference and the 1999 Annual Fall Meeting of the Biomedical Engineering Society. Proceedings of the First Joint BMES/EMBS Conference, 1999.

49. Litt B, Esteller R, D'Alessandro M, et al. Evolution of accumulated energy predicts seizures in mesial temporal lobe epilepsy. Engineering in Medicine and Biology, 1999. 21st Annual Conference and the 1999 Annual Fall Meeting of the Biomedical Engineering Society. Proceedings of the First Joint BMES/EMBS Conference, 1999.

50. Litt B, Esteller R, Echauz J, et al. Epileptic seizures may begin hours in advance of clinical onset: a report of five patients. Neuron 2001;30: 51-64.

51. Heck CN, King-Stephens D, Massey AD, et al. Two year seizure reduction in adults with medically intractable partial onset epilepsy treated with responsive neurostimulation: Final results of the RNS ${ }^{\circledR}$ System Pivotal trial. Epilepsia 2014;55:432-441.
52. The Vagus Nerve Stimulation Study Group. A randomized controlled trial of chronic vagus nerve stimulation for treatment of medically intractable seizures. Neurology 1995;45:224-230.

53. Boon P, Vonck K, Van Walleghem P, et al. Programmed and magnetinduced vagus nerve stimulation for refractory epilepsy. J Clin Neurophysiol 2001;18:402-407.

54. Morris GL, III. A retrospective analysis of the effects of magnetactivated stimulation in conjunction with vagus nerve stimulation therapy. Epilepsy Behav 2003;4:740-745.

55. Keilson MJ, Hauser WA, Magrill JP. Electrocardiographic changes during electrographic seizures. Arch Neurol 1989;46:1169-1170.

56. Smith PE, Howell SJ, Owen L, et al. Profiles of instant heart rate during partial seizures. Electroencephalogr Clin Neurophysiol 1989;72:207-217.

57. Zijlmans M, Flanagan D, Gotman J. Heart rate changes and ECG abnormalities during epileptic seizures: prevalence and definition of an objective clinical sign. Epilepsia 2002;43:847-854

58. Leutmezer F, Schernthaner C, Lurger S, et al. Electrocardiographic changes at the onset of epileptic seizures. Epilepsia 2003;44:348-354

59. van Elmpt WJ, Nijsen TM, Griep PA, et al. A model of heart rate changes to detect seizures in severe epilepsy. Seizure 2006;15:366375 .

60. Jeppesen J, Beniczky S, Fuglsang-Frederiksen A, et al. Detection of epileptic-seizures by means of power spectrum analysis of heart rate variability: a pilot study. Technol Health Care 2010;18: 417-426.

61. Shoeb A, Pang T, Guttag J, et al. Non-invasive computerized system for automatically initiating vagus nerve stimulation following patient-specific detection of seizures or epileptiform discharges. Int J Neural Syst 2009;19:157-172.

62. Osorio I. Automated seizure detection using ekg. Int J Neural Syst 2014;24:1450001

63. Boon P, Van Rijckevorsel K, Elger C, et al. Vagus nerve stimulation triggered by cardiac-based seizure detection, a prospective multicenter study. Epilepsy Curr 2014;14(Suppl.):21

64. Williams A, Gill S, Varma T, et al. Deep brain stimulation plus best medical therapy versus best medical therapy alone for advanced Parkinson's disease (PD SURG trial): a randomised, open-label trial. Lancet Neurol 2010;9:581-591.

65. Bronstein JM, Tagliati M, Alterman RL, et al. Deep brain stimulation for parkinson disease: An expert consensus and review of key issues. Arch Neurol 2011;68:165.

66. Pahwa R, Lyons KE, Wilkinson SB, et al. Long-term evaluation of deep brain stimulation of the thalamus. J Neurosurg 2006;104:506512.

67. Vidailhet M, Vercueil L, Houeto JL, et al. Bilateral, pallidal, deepbrain stimulation in primary generalised dystonia: a prospective 3 year follow-up study. Lancet Neurol 2007;6:223-229.

68. Carron R, Chaillet A, Filipchuk A, et al. Closing the loop of deep brain stimulation. Front Syst Neurosci 2013;7:112.

69. Little S, Brown P. What brain signals are suitable for feedback control of deep brain stimulation in Parkinson's disease? Ann N Y Acad Sci 2012;1265:9-24.

70. Feng XJ, Greenwald B, Rabitz H, et al. Toward closed-loop optimization of deep brain stimulation for Parkinson's disease: concepts and lessons from a computational model. J Neural Eng 2007;4: L14-L21.

71. Stypulkowski PH, Stanslaski SR, Denison TJ, et al. Chronic evaluation of a clinical system for deep brain stimulation and recording of neural network activity. Stereotact Funct Neurosurg 2013;91:220232.

72. Graupe D, Basu I, Tuninetti D, et al. Adaptively controlling deep brain stimulation in essential tremor patient via surface electromyography. Neurol Res 2010;32:899-904.

73. Basu I, Graupe D, Tuninetti D, et al. Pathological tremor prediction using surface electromyogram and acceleration: potential use in 'ON- 
OFF' demand driven deep brain stimulator design. J Neural Eng 2013;10:036019.

74. Yamamoto T, Katayama Y, Ushiba J, et al. On-demand control system for deep brain stimulation for treatment of intention tremor. Neuromodulation 2013;16:230-235.

75. Santaniello S, Fiengo G, Glielmo L, et al. Closed-loop control of deep brain stimulation: a simulation study [final version]. IEEE Trans Neural Syst Rehabil Eng 2011;19:15-24.

76. Kuhn AA, Kempf F, Brucke C, et al. High-frequency stimulation of the subthalamic nucleus suppresses oscillatory beta activity in patients with Parkinson's disease in parallel with improvement in motor performance. J Neurosci 2008;28:6165-6173.

77. Weinberger M, Mahant N, Hutchison WD, et al. Beta oscillatory activity in the subthalamic nucleus and its relation to dopaminergic response in Parkinson's disease. J Neurophysiol 2006;96:3248-3256.

78. Wingeier B, Tcheng T, Koop MM, et al. Intra-operative STN DBS attenuates the prominent beta rhythm in the STN in Parkinson's disease. Exp Neurol 2006;197:244-251.

79. Levy R, Ashby P, Hutchison WD, et al. Dependence of subthalamic nucleus oscillations on movement and dopamine in Parkinson's disease. Brain 2002;125:1196-1209.
80. Eusebio A, Thevathasan W, Doyle GL, et al. Deep brain stimulation can suppress pathological synchronisation in parkinsonian patients. J Neurol Neurosurg Psychiatry 2011;82:569-573.

81. Stanslaski S, Cong P, Carlson D, et al. An implantable Bidirectional brain-machine interface system for chronic neuroprosthesis research. Conf Proc IEEE Eng Med Biol Soc 2009;2009:54945497.

82. Stanslaski S, Afshar P, Cong P, et al. Design and Validation of a fully implantable, chronic, closed-loop neuromodulation device with concurrent sensing and stimulation. IEEE Trans Neural Syst Rehabil Eng 2012;20:410-21.

83. Stanford University, Medtronic. Neural signatures of Parkinson's disease (BrainRadio). Available at: http://clinicaltrials.gov/ct2/show/ NCT01990313. Accessed Feb 19, 2014.

84. Ward MP, Irazoqui PP. Evolving refractory major depressive disorder diagnostic and treatment paradigms: toward closed-loop therapeutics. Front Neuroeng 2010;3:7.

85. Okun MS, Foote KD, Wu SS, et al. A trial of scheduled deep brain stimulation for Tourette syndrome: moving away from continuous deep brain stimulation paradigms. JAMA Neurol 2013;70: 85-94. 